\title{
TWO CONVOLUTION TRANSFORMS WHICH ARE INVERTED BY CONVOLUTIONS
}

\author{
D. V. WIDDER
}

1. Introduction. The inversions of two convolution transforms are considered here and sufficient conditions are stated for the two inversions.

The first inversion, due to R. G. Buschman [2], is

$$
\begin{aligned}
& f(x)=\int_{x}^{1} P_{n}\left(\frac{t}{x}\right) g(t) d t, \\
& g(x)=\int_{x}^{1} P_{n-2}\left(\frac{x}{t}\right) h(t) d t, \\
& h(t)=t^{1-n} \frac{d}{d t}\left\{\frac{1}{t} \frac{d}{d t} t^{n} f(t)\right\} .
\end{aligned}
$$

Here $P_{n}(t)$ is the Legendre polynomial of degree $n=2,3, \cdots$, and it is assumed that $g(1)=0$ (whence $f(1)=f^{\prime}(1)=0$ ).

The other, due to $\mathrm{Ta} \mathrm{Li}[3]$ is given by the pair

$$
\begin{aligned}
& f(x)=\int_{x}^{1} \frac{T_{n}\left(\frac{t}{x}\right)}{\sqrt{ }\left(t^{2}-x^{2}\right)} g(t) d t, \\
& g(x)=\frac{-2}{\pi} \int_{x}^{1} \frac{T_{n-1}\left(\frac{x}{t}\right)}{\sqrt{ }\left(t^{2}-x^{2}\right)} t^{1-n} \frac{d}{d t}\left\{t^{n} f(t)\right\} d t .
\end{aligned}
$$

Here $T_{n}(x)$ is the Tchebichef polynomial

$$
T_{n}(x)=\cos \left(n \cos ^{-1} x\right), \quad n=1,2, \cdots .
$$

It is proposed to give new proofs of these formulas. The method will be the natural one involving Laplace transforms. It is hoped that the analytic character of the generating functions involved will reveal what is really behind the inversions.

2. Operational considerations. From the usual definition of the operator $\exp (a D) f(x)$ as $f(x+a)$, it is clear that there is a ready interpretation of the operator $M(D)$ whenever $M(s)$ is the generating function of a Laplace integral:

Received by the editors August 6, 1962. 


$$
M(s)=\int_{-\infty}^{\infty} e^{-s t} K(t) d t
$$

Thus

$$
\begin{aligned}
M(D) g(x) & =\int_{-\infty}^{\infty} e^{-t D} g(x) K(t) d t \\
& =\int_{-\infty}^{\infty} g(x-t) K(t) d t=g * K,
\end{aligned}
$$

and we obtain the symbolic inversion

$$
\frac{1}{M(D)} g * K=g \text {. }
$$

However, suitable interpretation of the operator $1 / M(D)$ is not always evident. In [4] the author considered a large class of meromorphic functions $M(s)$ for which the formula (2.3) was meaningful, and applied the result to the familiar sine-transform when $M(s)$ $=\Gamma(s) \sin (\pi s / 2)$.

For the purposes of the present note we need to assume that $K(x)$ and $g(x)$ vanish for negative $x$, so that (2.1) becomes

$$
M(s)=\int_{0}^{\infty} e^{-s t} K(t) d t
$$

and the convolution (2.2) becomes

$$
\int_{0}^{x} K(x-t) g(t) d t
$$

Since every unilateral transform (2.4) vanishes at infinity, it is clear that $1 / M(s)$ cannot be a generating function. Hence in this case $1 / M(D)$ cannot be interpreted as a convolution. It is for this reason that the inversions of Buschman and $\mathrm{Li}$, which do involve convolutions, are particularly interesting. But note that in both cases the convolutions do not bear on $f(x)$ itself but on some linear combination of $f(x)$ and its derivatives.

3. An analogous simple example. To illustrate the remarks of the previous section, consider the transform

$$
f(x)=\frac{1}{\sqrt{ } \pi} \int_{0}^{x} \frac{g(t)}{\sqrt{ }(x-t)} d t .
$$


Equations (2.2) and (2.3) become

$$
\begin{aligned}
& \frac{1}{\sqrt{ } D} g(x)=\frac{1}{\sqrt{ } \pi} \int_{0}^{x} \frac{g(t)}{\sqrt{ }(x-t)} d t=f(x), \\
& \sqrt{ } D f(x)=g(x) .
\end{aligned}
$$

As predicted, $\sqrt{ } s$ is not a generating function, but if we write it as $s / \sqrt{ } s$, then (2.3) becomes

$$
\begin{aligned}
D \frac{1}{\sqrt{ } D} f(x) & =g(x), \\
\frac{d}{d x} \frac{1}{\sqrt{ } \pi} \int_{0}^{x} \frac{f(t)}{\sqrt{ }(x-t)} d t & =g(x) .
\end{aligned}
$$

Of course (3.1) and (3.2) are the familiar definitions of a fractional integral and a fractional derivative, respectively, of order 1/2. An integration by parts yields

$$
g(x)=\frac{1}{\sqrt{ } \pi} \int_{0}^{x} \frac{f^{\prime}(t)}{\sqrt{ }(x-t)} d t,
$$

and the resemblance to Ta Li's formula is evident.

4. The Legendre kernel. Obvious changes of variables reduce (1.1), (1.2) to

$$
\begin{aligned}
& f\left(e^{-x}\right)=\int_{0}^{x} P_{n}\left(e^{x-t}\right) g\left(e^{-t}\right) e^{-t} d t, \\
& g\left(e^{-x}\right)=\int_{0}^{x} P_{n-2}\left(e^{-x+t}\right) h\left(e^{-t}\right) e^{-t} d t .
\end{aligned}
$$

The generating functions for both kernels are known:

$$
\begin{aligned}
& M_{n}(s)=\int_{0}^{\infty} e^{-s t} P_{n}\left(e^{t}\right) d t=\frac{1}{s-n} \prod_{k=1}^{n-1} \frac{s+k}{s-n+2 k}, \\
& N_{n}(s)=\int_{0}^{\infty} e^{-s t} P_{n}\left(e^{-t}\right) d t=\frac{1}{s+n} \prod_{k=1}^{n-1} \frac{s-k}{s+n-2 k} .
\end{aligned}
$$

The second of these is formula (3) on p. 171 of [1], and the first is easily derived therefrom.

The transform of $(4.1)$ is

$$
F(s)=M_{n}(s) G(s+1),
$$

where $F(s)$ and $G(s)$ are the Laplace transforms of $f\left(e^{-t}\right)$ and $g\left(e^{-t}\right)$, respectively. 
1963] TWO CONVOLUTION TRANSFORMS INVERTED BY CONVOLUTIONS 815

To transform (4.2), we need to compute

$$
\int_{0}^{\infty} e^{-s t} h\left(e^{-t}\right) e^{-t} d t=\int_{0}^{1} y^{s} h(y) d y .
$$

Using the definition of $h(y)$, the fact that $f(1)=f^{\prime}(1)=0$, and integration by parts, this integral becomes

$$
\begin{aligned}
(s-n+1)(s-n-1) \int_{0}^{1} f(y) y^{s-2} d y & \\
= & (s-n+1)(s-n-1) F(s-1) .
\end{aligned}
$$

Thus the transform of (4.2) is

$$
G(s)=(s-n+1)(s-n-1) N_{n-2}(s) F(s-1) .
$$

Consequently our inversion will be established if

$$
M_{n}(s-1) N_{n-2}(s)(s-n+1)(s-n-1)=1 .
$$

If we insert the products (4.3), (4.4) in the left side of this equation and multiply numerator and denominator by $(s-n)(s-n-2)$, each becomes

$$
\prod_{k=-n-1}^{n-2}\left(s_{i}^{-}+k\right)
$$

so that (4.5) is an identity.

5. Two Laplace transforms. To establish (1.4), we shall need the following lemma.

LEMMA.

$$
\begin{aligned}
M_{n}(s) & =\int_{0}^{\infty} e^{-s t} \frac{T_{n}\left(e^{t}\right)}{\sqrt{ }\left(1-e^{-2 t}\right)} d t=2^{s-2} B\left(\frac{s+n}{2}, \frac{s-n}{2}\right) \\
N_{n}(s) & =\int_{0}^{\infty} e^{-s t} \frac{T_{n}\left(e^{-t}\right)}{\sqrt{ }\left(1-e^{-2 t}\right)} d t \\
& =\pi /\left\{s 2^{s} B\left(\frac{s+n+1}{2}, \frac{s-n+1}{2}\right)\right\}
\end{aligned}
$$

Here

$$
B(x, y)=\frac{\Gamma(x) \Gamma(y)}{\Gamma(x+y)} .
$$


From the familiar recursion formula

$$
T_{n+1}(t)=2 t T_{n}(t)-T_{n-1}(t)
$$

it follows that

$$
\begin{aligned}
& M_{n+1}(s)=2 M_{n}(s-1)-M_{n-1}(s), \\
& N_{n+1}(s)=2 N_{n}(s+1)-N_{n-1}(s) .
\end{aligned}
$$

Now it is easily verified that the functions on the right of (5.1), (5.2) satisfy (5.3), (5.4), respectively. Moreover, (5.1) and (5.2) are seen to be valid for $n=0,1$ by elementary methods. Hence the lemma is established. Equation (5.2) may be checked by formula (2) on p. 324 of $[1]$.

6. The Tchebichef kernel. The pair (1.3), (1.4) is equivalent to

$$
\begin{aligned}
& f\left(e^{-x}\right)=\int_{0}^{x} \frac{T_{n}\left(e^{x-t}\right)}{\sqrt{ }\left(1-e^{-2(x-t)}\right)} g\left(e^{-t}\right) d t \\
& g\left(e^{-x}\right)=-\frac{2}{\pi} \int_{0}^{x} \frac{T_{n-1}\left(e^{t-x}\right)}{\sqrt{ }\left(1-e^{-2(x-t)}\right)}\left[n f\left(e^{-t}\right)+e^{-t} f^{\prime}\left(e^{-t}\right)\right] d t .
\end{aligned}
$$

Again defining $F(s)$ and $G(s)$ as the Laplace transforms of $f\left(e^{-t}\right)$ and $g\left(e^{-t}\right)$, respectively, we see that $(6.1)$ becomes

Since

$$
F(s)=M_{n}(s) G(s) \text {. }
$$

$$
\int_{0}^{\infty} e^{-s t}\left[n f\left(e^{-t}\right)+e^{-t} f^{\prime}\left(e^{-t}\right)\right] d t=n F(s)-s F(s),
$$

(6.2) becomes

$$
G(s)=\frac{2}{\pi}(s-n) N_{n-1}(s) F(s) .
$$

To establish (6.2) we need only show that

$$
\frac{2}{\pi} M_{n}(s) N_{n-1}(s)(s-n)=1 .
$$

By inserting the values of $M_{n}(s)$ and $N_{n-1}(s)$, as given by the lemma, and by using the functional equation for $\Gamma(s)$, this is easily seen to be an identity.

7. Conclusions. The key to the pair (1.1), (1.2) is the fact that the meromorphic function $M_{n}(s)$ is rational, the quotient of a polynomial of degree $n-1$ by another of degree $n$. Its reciprocal thus becomes a 
generating function when divided by a polynomial of degree two. This degree is mirrored in the fact that $h(t)$ is defined by a differential operator of order two. Of course the fact that the inversion kernel is again a Legendre polynomial is a result of the special nature of the function (4.3). In the case of the pair (1.3), (1.4), $M_{n}(s)$ has infinitely many poles and infinitely many zeros. Its reciprocal becomes a generating function after division by the linear function $(s-n)$. Correspondingly, the differential operator appearing in (1.4) is of first order.

In conclusion let us state as theorems sufficient conditions for the two inversions. We convert the results to the more familiar convolution transform.

THEOREM 1. If

1. $g(x) \in C^{1}, 0 \leqq x<\infty, g(0)=0$,

2. $f(x)=\int_{0}^{x} P_{n}\left(e^{x-t}\right) g(t) d t$, then

A. $f(x) \in C^{2}, 0 \leqq x<\infty, f(0)=f^{\prime}(0)=0$,

B. $g(x)=\int_{0}^{x} P_{n-2}\left(e^{t-x}\right) e^{t-x} h(t) d t$,

$$
h(t)=e^{(n-2) t} \frac{d}{d t} e^{2 t} \frac{d}{d t}\left[e^{-n t} f(t)\right] .
$$

THEOREM 2. If

1. $g(x) \in C, 0 \leqq x<\infty$,

2. $f(x)=\int_{0}^{x} T_{n}\left(e^{x-t}\right)\left(1-e^{-2(x-t)}\right)^{-1 / 2} g(t) d t$, then

A. $f(x) \in C^{1}, 0 \leqq x<\infty, f(0)=0$,

B. $g(x)=(2 / \pi) \int_{0}^{x} T_{n-1}\left(e^{t-x}\right)\left(1-e^{-2(x-t)}\right)^{-1 / 2} h(t) d t$,

$$
h(t)=e^{n t} \frac{d}{d t}\left[e^{-n t} f(t)\right] .
$$

\section{REFERENCES}

1. A. Erdélyi, W. Magnus, F. Oberhettinger, and F. G. Tricomi, Tables of integral transforms, Vol. II, McGraw-Hill, New York, 1954.

2. R. G. Buschman, An inversion integral for a Legendre transformation, Amer. Math. Monthly 69 (1962), 288-289.

3. $\mathrm{Ta} \mathrm{Li}, A$ new class of integral transforms, Proc. Amer. Math. Soc. 11 (1960), 290-298.

4. D. V. Widder, Symbolic inversions of the Fourier-sine transforms and of related transforms, J. Indian Math. Soc. 14 (1950), 119-128.

HARVARD UNIVERSITY 